\title{
ARNE RADTKE-DELACOR
}

\section{DIE »GELENKTE WIRTSCHAFT« IN FRANKREICH}

\author{
Versuch einer vergleichenden Untersuchung \\ der technokratischen Strukturen der NS-Besatzungsmacht \\ und des Vichy-Regimes (1940-1944)
}

Staatsinterventionismus und Wirtschaftslenkung erreichten sowohl in Frankreich als auch in Deutschland während des Zweiten Weltkrieges ein bis dahin nicht gekanntes Ausmaß, dessen offenkundiger Ausdruck der Machtzuwachs technokratischer Funktionseliten ${ }^{1}$ in beiden Ländern war. Bislang blieb die Untersuchung der Ziele, Akteure, Methoden und Ergebnisse des Wirtschaftsdirigismus Vichys ${ }^{2}$ und der NS-Kriegswirtschaft ${ }^{3}$ vorwiegend Gegenstand nationalgeschichtlicher Zugänge, so daß es vor allem an vergleichenden systemanalytischen Untersuchungen zu den ökonomischen Strukturen und technokratischen Eliten beider Länder in dieser Zeit fehlt. Der vorliegende Beitrag kann diese Lücke bei weitem nicht schließen, stellt aber einen Versuch dar, anhand der vierjährigen Interaktion von Besatzungs- und Vichy-Regime die in Frankreich etablierten technokratischen Strukturen beider Seiten einer komparativen Analyse zu unterziehen und hierbei nicht nur nach den sozialen Bindungen und ideologischen Prägungen der Hauptakteure der "gelenkten Wirtschaft" (économie dirigée), sondern auch nach deren Herrschaftspraxis, insbesondere im Kontext der Kollaborationspolitik, zu fragen. Diese Untersuchung ist aber nicht nur statisch im Sinne einer vergleichenden Gegenüberstellung beider Regime, sondern auch dynamisch als Analyse ihrer wechselseitigen Beeinflussung im Laufe der Besatzungszeit zu verstehen, die sich vor allem auf dem Feld der Wirtschaftspolitik manifestierte.

\footnotetext{
${ }^{1}$ Der Terminus »Technokraten« war damals noch nicht geläufig. Trotz seines Anachronismus beschreibt er in seiner heutigen Konnotation zutreffend die damalige soziale und politische Funktion staatlicher und ökonomischer Eliten. Siehe Gérard BRUN, Technocrates et technocratie en France 1918-1945, Paris 1985.

${ }^{2}$ Michel Margairaz, L'État, les finances et l'économie. Histoire d'une conversion 19321952, 2 Bde, Paris 1991, bes. Bd. I, S. 508-712.

${ }^{3}$ Dietrich EichHOLTZ, Geschichte der deutschen Kriegswirtschaft 1939-1945, 3 Bde, Berlin 1969-1996. Eichholtz beschränkt sich in seinem monumentalen Standardwerk nicht nur auf Deutschland, sondem ist bemüht, die Okkupationswirtschaft des gesamten deutschen Machtbereichs in die Analyse einzubeziehen.
} 
Die Analyse beginnt daher mit einer einleitenden Untersuchung der spezifischen Formen der strategischen Anpassung der deutschen Militäradministration an die Besonderheiten der Verhältnisse in Frankreich im Zuge des Waffenstillstands von 1940 sowie der sozialstrukturellen und institutionellen Realität der NS-Polykratie im besetzten Gebiet. Danach wird der Frage nach der tatsächlichen Rolle der Technokraten innerhalb des Vichy-Regimes und nach der Bedeutung wirtschaftlicher Globalsteuerung in der Programmatik der umfassenden Reorganisation des französischen Staatsapparates nachgegangen. Ferner ist nach den Sozialisations- und Herrschaftserfahrungen der technokratischen Elite Frankreichs sowie der Rolle ideologischer und pragmatischer Faktoren ihrer Kollaborationspolitik zu fragen. Hierin wird auch der singuläre Fall des Mustertechnokraten des Regimes, Jean Bichelonne, einbezogen, in dem sich ein hohes $\mathrm{Ma} ß$ an Intelligenz mit einer ausgeprägten politischen Radikalität verband. Nach der Untersuchung der deutsch-französischen Interessenkonvergenzen als wesentlicher Faktor der gegenseitigen Beeinflussung der beiden Regime wird schließlich die reale Funktion der Wirtschaftskollaboration in den Augen der Technokraten der NS-Okkupationsverwaltung analysiert.

Die Errichtung der deutschen Militäradministration im Pariser Hotel Majestic im Sommer 1940: „Aufsichtsverwaltung« als strategische Anpassung an den Sonderfall Frankreich

Die organisatorischen Aporien und strukturellen Antagonismen des deutschen Besatzungsregimes, die sich in der Vielzahl rivalisierender ziviler wie militärischer Dienststellen und Sonderorganisationen in den besetzten Gebieten, vor allem auf dem Sektor der kriegswirtschaftlichen Ausbeutung fremder Ressourcen, manifestierten, sind signifikante Strukturmerkmale der NS-Diktatur ${ }^{4}$. Tatsächlich lag das Ziel der NS-Führung nicht in der Vereinheitlichung der Besatzungsverwaltung und der Herrschaftsmethoden, "vielmehr stellte man sich auf eine Unterschiedlichkeit ein, die die eigentlichen Absichten verschlei-

\footnotetext{
${ }^{4}$ Auf das »Kompetenzchaos« und die »polykratischen« Züge des Regimes als Interpretationsmuster stützt sich vor allem der "funktionalistische" Ansatz in der Historiographie zum »Dritten Reich«. Siehe namentlich die grundlegenden Beiträge von Peter HÜTTENBERGER, Nationalsozialistische Polykratie, in: Geschichte und Gesellschaft. Zeitschrift für Historische Sozialwissenschaft 2 (1976) S. 417-442 und Martin BROSZAT, Der Staat Hitlers. Grundlegung und Entwicklung seiner inneren Verfassung, München ${ }^{14} 1995$ (dtv-Weltgeschichte des 20. Jahrhunderts, 9), S. 363-395, bes. S. 375-379. Zur NS-Verwaltungspolitik allgemein: Dieter REBENTISCH, Führerstaat und Verwaltung im Zweiten Weltkrieg. Verfassungsentwicklung und Verwaltungspolitik 1939-1945, Stuttgart 1989 (Frankfurter Historische Abhandlungen, 29).
} 
erte und die Interessen der Okkupanten so besser absicherte $" ~ "$. Unter den beteiligten Institutionen besaß aufgrund ihrer administrativen Funktion und ökonomischen Kompetenz die Wirtschaftsabteilung des Militärbefehlshabers in Frankreich eine Schlüsselstellung. Der Amtssitz des Militärbefehlshabers im Pariser Hotel Majestic wurde zum Synonym für die deutsche Besatzungsherrschaft. Durch die Übertragung der vollziehenden Gewalt im besetzten Gebiet auf den Militärbefehlshaber ${ }^{6}$ besaß das Majestic in der NS-Besatzungswirtschaft in Frankreich entscheidende hoheitliche Aufgaben. Nach Artikel 3 des Waffenstillstandsvertrags vom 22. Juni 1940 übten die deutschen Militärbefehlshaber in den okkupierten Teilen Frankreichs »alle Rechte der besetzenden Macht« aus $^{7}$, eine bewußt vage gehaltene Formel, um eine über die Haager Landkriegsordnung (HLKO) ${ }^{8}$ hinausgehende Exekutivgewalt der Besatzungsverwaltung, insbesondere auf wirtschaftlichem Gebiet, zu konstruieren?

Die Militäradministration war von vornherein als "Aufsichtsverwaltung" konzipiert, eine zeitgenössische Begriffsschöpfung der Besatzungsstäbe ${ }^{10}$. Sie implizierte den Grundgedanken, eine Belastung der Okkupationsorgane mit der alleinigen Verwaltung des besetzten Gebiets nach Möglichkeit zu vermeiden. Neben dem Vorteil der Einsparung personeller Ressourcen verbarg sich dahinter das Kalkül, die im Waffenstillstandsabkommen zugebilligte französische Verwaltungshoheit über das gesamte französische Staatsgebiet (also nicht nur der unbesetzten Zone) für eigene okkupationspolitische Ziele zu funktio-

${ }^{5}$ Czesław MADAJCZYK, Chaos, Systemhaftigkeit oder Systeme? Das Dritte Reich in der Phase der militärischen Expansion, in: Ralph MELVILlE, Claus SCHARF u.a. (Hg.), Deutschland und Europa in der Neuzeit. FS Karl Otmar Freiherr von Aretin, Stuttgart 1988 (Veröffentlichungen des Instituts für Europäische Geschichte Mainz, Abt. Universalgeschichte, 134, 2. Halbband), S. 931-954, hier S. 940.

${ }^{6}$ Die vollziehende Gewalt bezog sich nicht nur auf die Exekutive, sondern auch auf die Legislative (Verordnungen der Militärverwaltung) und Jurisdiktion (Militärgerichtsbarkeit). Vgl. Hermann BÖHME, Der deutsch-französische Waffenstillstand im Zweiten Weltkrieg. Erster Teil: Entstehung und Grundlagen des Waffenstillstandes von 1940, Stuttgart 1966 (Quellen und Darstellungen zur Zeitgeschichte, 12), S. 160. Territorial blieben die beiden nordfranzösischen Departements Nord und Pas-de-Calais (unter dem Militärbefehlshaber in Belgien und Nordfrankreich) und die drei ostfranzösischen Departements Haut-Rhin, BasRhin und Moselle (unter den Chefs der Zivilverwaltung) von der Befehlsgewalt des Militärbefehlshabers in Frankreich (MBF) ausgenommen.

${ }^{7}$ Akten zur deutschen auswärtigen Politik 1918-1945. Aus dem Archiv des Auswärtigen Amtes (ADAP), Serie D, Bd. IX, S. 555 (Dok. 523).

${ }^{8}$ Convention concernant les lois et coutumes de la guerre sur terre. Den Haag, 18. Oktober 1907, in: Reichsgesetzblatt, Teil I (RGB1 I) 1910, S. 107-151.

${ }^{9}$ Auf die HLKO wurde im Vertragstext nirgendwo Bezug genommen. BÖHME, Waffenstillstand, S. 158 weist in diesem Zusammenhang darauf hin, Hitler habe in dem vom Oberkommando der Wehrmacht (OKW) gefertigten Entwurf des Waffenstillstandsvertrages die explizite Bezugnahme auf die HLKO gestrichen und durch den Begriff der »Rechte der besetzenden Macht« ersetzt.

${ }^{10}$ Siehe Werner BEST, Großraumordnung und Großraumverwaltung, in: Zeitschrift für Politik 32 (1942) S. 406-412, hier S. 408f. 
nalisieren, in der Hoffnung, die Akzeptanz deutscher Verordnungen in der französischen Bevölkerung durch Zwischenschaltung der landeseigenen Verwaltung zu steigern. Nach Artikel 3 des Waffenstillstandsvertrages waren alle französischen Dienststellen durch ihre Regierung anzuweisen, die Anordnungen der Militärverwaltung »mit allen Mitteln« zu unterstützen und »in korrekter Weise « mit ihr zusammenzuarbeiten ${ }^{11}$. Umgekehrt war auch die Militärverwaltung prinzipiell verpflichtet, die französischen Behörden bei der Durchführung ihrer Aufgaben heranzuziehen ${ }^{12}$, wobei nach den internen Richtlinien über die Ausübung des Aufsichts- und Weisungsrechts gegenüber den französischen Behörden vom August 1940 hiervon ausgiebig Gebrauch gemacht werden sollte ${ }^{13}$.

Mit der Einarbeitung der Besatzungsstäbe in die spezifischen Verhältnisse Frankreichs ${ }^{14}$ wurden vom Leiter der Abteilung Verwaltung im Verwaltungsstab des Militärbefehlshabers ${ }^{15}$, SS-Brigadeführer Werner Best, die okkupationspolitischen Strategien der "Aufsichtsverwaltung « zu einer über Frankreich weit hinausgreifenden Systematik zusammengefaßt und verfeinert. Bis Juni 1940 Stellvertreter Heydrichs als Leiter des Amtes I des Reichssicherheitshauptamts, zählte Best, ein idealtypischer Exponent der "Generation des Unbedingten ${ }^{16}$, zu den zentralen Figuren der Vordenker einer durchrationalisierten NS-»Großraumverwaltung" Westeuropas ${ }^{17}$. Der Terminus »Aufsichtsver-

$"$ ADAP, Serie D, Bd. IX, S. 555 (Dok. 523).

${ }^{12}$ Bundesarchiv (BA) Berlin, R 43 II/675, fol. 76-78: Erlaß (GenStdH/GenQu Nr. 15530/40) des Oberbefehlshabers des Heeres $(\mathrm{ObdH})$ betr. Militärverwaltung in den besetzten französischen Gebieten. Hauptquartier des Oberkommandos des Heeres (OKH), 26. Juni 1940, gez. von Brauchitsch.

13 Bundesarchiv-Militärarchiv, Freiburg (BA-MA), RW 35/4, fol. 50-55: Aufzeichnung (ohne Unterschrift) des Verwaltungsstabes des Chefs der Militärverwaltung in Frankreich betr. Richtlinien für die Ausübung der Aufsicht über die französische Verwaltung im besetzten Gebiet durch die deutsche Militärverwaltung in Frankreich. Paris, 26. August 1940, $10 \mathrm{~S}$. (Anlage A zum geheimen Lagebericht des Verwaltungsstabes des Chefs der Militärverwaltung in Frankreich für den Monat August 1940).

${ }^{14}$ Im Gegensatz zu allen anderen eroberten Ländern blieb bis November 1942 ein Teil Frankreichs unter einer - zumindest nominell - souveränen Regienung, die den größten Teil des Kolonialreichs und der Flotte kontrollierte, unbesetzt.

is Zur Organisationsstruktur der Militärverwaltung siehe Hans UMBREIT, Der Militärbefehlshaber in Frankreich.1940-1944, Boppard 1968 (Wehrwissenschaftliche Forschungen, Abt. Militärgeschichtliche Studien, 7).

${ }^{16}$ So der prägnante Titel der grundlegenden Untersuchung von Michael WILDT, Generation des Unbedingten. Das Führungskorps des Reichssicherheitshauptamtes, Hamburg 2002.

${ }^{17}$ Die Schlüsselrolle Bests hinsichtlich der konzeptionell-administrativen Vorbereitung des NS-»Großraums « ist in der zeitgeschichtlichen Forschung lange Zeit nicht erkannt worden. Siehe jetzt Ulrich HERBERT, Best. Biographische Studien über Radikalismus, Weltanschauung und Vernunft, 1903-1989, Bonn 1996, bes. S. 251-322; Ahlrich MEYER, Großraumpolitik und Kollaboration im Westen. Werner Best, die Zeitschrift "Reich - Volksordnung Lebensraum « und die deutsche Militärverwaltung in Frankreich 1940-42, in: Beiträge zur nationalsozialistischen Gesundheits- und Sozialpolitik 10 (1992) S. 29-76. 
waltung «, mit dem er seine Konzeption der Militärverwaltung in Frankreich definierte $^{18}$, prägte in analytischer Differenzierung der Formen nationalsozialistischer Besatzungsherrschaft den amtlichen und (verwaltungs-)wissenschaftlichen Sprachgebrauch über das Kriegsende hinaus ${ }^{19}$.

Die politischen und wirtschaftlichen Aufgaben der deutschen Militärverwaltung in Frankreich waren nach Best nicht auf den unmittelbaren Besatzungszweck beschränkt, sondern explizit im Hinblick auf die "zu schaffende europäische Großraumordnung « zu definieren. In diesem Sinne sollten die Besatzungserfahrungen des »französischen Experiments« zur »Schaffung und zum Erfolg einer künftigen deutschen Großraum-Verwaltung" operationalisiert werden ${ }^{20}$. Hinter der "Aufsichtsverwaltung" in Frankreich stand ein Programm, das nach außen hin unter formaler Beachtung besonders des Artikels 43 der $\mathrm{HLKO}^{21}$ die einheimische Verwaltung und Gesetzgebung möglichst unangetastet ließ, tatsächlich aber als Vorstufe einer europäischen Nachkriegsordnung galt, in der auch nach Best "Vernichtung und Verdrängung fremden Volkstums« zur gängigen Herrschaftspraxis zählen sollte ${ }^{22}$. Mit ihr war die geeignete Formel gefunden, die langfristigen politischen Implikationen der NS-Besatzungsherrschaft durch die propagandawirksame $»$ Korrektheit« des »Experiments« der Militärverwaltung in Frankreich zu kaschieren.

\section{Die Personalstruktur des Majestic: die Bedeutung der Technokraten}

Gemäß der Maxime, walle notwendigen Zwecke mit dem geringsten $\mathrm{Maß}$ an Aufsichtstätigkeit zu verwirklichen ${ }^{23}$, kam der Verwaltungsstab des Militärbefehlshabers zunächst mit einer Personalstärke von rund 1500 Militärverwaltungsbeamten zur administrativen Kontrolle über drei Fünftel des französi-

\footnotetext{
${ }^{18}$ BEST, Großraumordnung, S. 408f.

${ }^{19}$ Auch von Eberhard JÄCKEL, Frankreich in Hitlers Europa. Die deutsche Frankreichpolitik im Zweiten Weltkrieg, Stuttgart 1966 (Quellen und Darstellungen zur Zeitgeschichte, 14), S. 63 und UMBREIT, Militärbefehlshaber, S. 27 wurde Bests Begriffsschöpfung unreflektiert übernommen.

${ }^{20}$ Werner BEST, Die deutsche Militärverwaltung in Frankreich, in: Reich - Volksordnung Lebensraum. Zeitschrift für völkische Verfassung und Verwaltung 1 (1941) S. 29-76, hier S. $42,47,74$ u. 76.

${ }^{21}$ Artikel 42 bis 56 der Haager Konvention regelten die "Militärische Gewalt auf besetztem feindlichen Gebiete«. Siehe RGB1 I, 1910, S. 147-151.

22 BEST, Großraumordnung, S. 407: »Vernichtung und Verdrängung fremden Volkstums widerspricht nach geschichtlichen Erfahrungen den Lebensgesetzen nicht, wenn sie vollständig geschieht. [...] Alle Völker, die fremdvölkische Sklaven und Heloten verwenden, sterben durch Vermischung den Rassetod «.

${ }^{23}$ DERS., Militärverwaltung, S. 74.
} 
schen Staatsgebiets mit über 20 Millionen Einwohnern aus. Eine geringere deutsche Verwaltungsdichte gab es im besetzten Europa nur noch in Dänemark (89 Beamte auf 3,8 Millionen Einwohner) ${ }^{24}$. Allein ein Drittel des gesamten Verwaltungspersonals entfiel auf die Pariser Zentrale im Hotel Majestic. Mit den Ende 1941 einsetzenden Abgaben nach dem Osten verringerte sich laufend die Personalstärke, die sich gegen Ende der Besatzungszeit im Sommer 1944 für das gesamte französische Staatsgebiet (ohne Elsaß-Lothringen) einschließlich der Kanalinseln trotz Totalbesetzung des Landes und progressiv steigender Aufgaben und Schwierigkeiten auf nur noch 1180 Beamte, darunter 595 höhere Beamte belief ${ }^{25}$. Der ungewöhnlich hohe Anteil höherer Verwaltungsspezialisten, die allein rund die Hälfte des gesamten Apparates stellten, war durch die besondere Besatzungsform der "Aufsichtsverwaltung" bedingt. Ihre Sozialisation war keineswegs kongruent. Ein nicht unerheblicher Teil von ihnen kam aus der Wirtschaft selbst (Techniker und Kaufleute). Das Personal der Militärverwaltung setzte sich lediglich zu etwa zwei Dritteln aus hauptamtlichen Verwaltungsbeamten zusammen, $20 \%$ entfielen auf die verschiedenen Wirtschaftsorganisationen wie die Reichswirtschaftskammer oder die Reichs- und Wirtschaftsgruppen. Die restlichen $15 \%$ entstammten freien Berufen oder der Privatwirtschaft ${ }^{26}$. Diese Fachleute waren keine Beamte und fanden entsprechend ihrem militärischen Dienstgrad als Reserveoffiziere Verwendung.

Mit der Dauer des Krieges nahm die Bedeutung der Wirtschaftsabteilung der Militärverwaltung kontinuierlich zu, so daß deren Leiter, Kriegsverwaltungschef Elmar Michel, am 1. August 1942 unter Beibehaltung seiner bisherigen Funktionen zum Chef des gesamten Verwaltungsstabes avancierte. Michel, Ministerialdirigent im Reichswirtschaftsministerium, fungierte dort als Leiter der Abteilung $» S «$ (Sonderabteilung) ${ }^{27}$ und wurde in seiner Eigenschaft als Spezialist für Rohstoffbewirtschaftung und »wehrwirtschaftliche Fragen« kurz nach dem Waffenstillstand auf Vorschlag des Reichswirtschaftsministers Walther Funk vom Beauftragten für den Vierjahresplan, Hermann Göring,

${ }^{24}$ BA Koblenz, N 1023/3 [unfoliiert]: "Die deutschen Aufsichtsverwaltungen in Frankreich, Belgien, den Niederlanden, Norwegen, Dänemark und im Protektorat Böhmen und Mähren. Vergleichende Übersicht«. (Denkschrift des Ministerialdirektors und Kriegsverwaltungschefs Werner Best für das OKH), o.D. [September 1941], 72 S., hier S. 15.

${ }^{25}$ BA-MA, RW 35/244, fol. 1-70: »Allgemeiner Rückblick« (Schlußbericht der Militärverwaltung in Frankreich 1940-1944), o.D. [Potsdam, 25. März 1945], 2. Ausfertigung, 69 S., bes. fol. 6. Der unsignierte Schlußbericht wurde vermutlich von Elmar Michel verfaßt.

${ }^{26}$ Ibid.

${ }^{27}$ Vgl. die Organisationsübersicht des Reichswirtschaftsministeriums (RWM) in Rolf-Dieter MüLLER, Die Mobilisierung der deutschen Wirtschaft für Hitlers Kriegführung, in: Das Deutsche Reich und der Zweite Weltkrieg, Bd. 5: Organisation und Mobilisierung des deutschen Machtbereichs, 1. Halbband: Kriegsverwaltung, Wirtschaft und personelle Ressourcen 1939-1941, hg. vom Militärgeschichtlichen Forschungsamt, Stuttgart 1988 (Beiträge zur Militär- und Kriegsgeschichte), S. 445. 
zum Leiter der Wirtschaftsabteilung beim Chef der Militärverwaltung in Frankreich emannt ${ }^{28}$. Es handelte sich um einen außergewöhnlich erfahrenen Fachbeamten konservativer Prägung, der wegen seiner umfassenden Wirtschaftskenntnisse und seines pragmatischen Verhandlungsstils ${ }^{29}$ geradezu prädestiniert schien, die an ihn regelmäßig herangetragenen wirtschaftspolitischen Forderungen des Vierjahresplans auch in Frankreich gegenüber der VichyAdministration und Skeptikern in den eigenen Reihen durchzusetzen. Der Aufstieg Michels zum Chef des Verwaltungsstabes zeigt zudem »die Bedeutung, die der Wirtschaftsabteilung innerhalb der Militärverwaltung inzwischen zukam, nachdem die deutsch-französische scollaboratione sich vor allem auf den wirtschaftlichen Sektor verlagert hatte [...] und Frankreich für Deutschland vornehmlich als auszubeutendes Gebiet von Interesse war ${ }^{30}$. Michel unterstand ein Stab von Gruppenleitern für die jeweiligen wirtschafts- und finanzpolitischen Fachgebiete ${ }^{31}$, die in ihrer Mehrheit der Sonderverwaltung des Generalbevollmächtigten für die Wirtschaft entstammten ${ }^{32}$ und sich bereits durch ihre aktive Beteiligung an den Planungen zur Errichtung der Okkupationsverwaltung in den zu besetzenden Westgebieten für ihre spätere Aufgabenstellung profiliert hatten ${ }^{33}$.

28 BA-MA, RW 19/Wi I A3/94(a), fol. 71: Der Beauftragte für den Vierjahresplan (V.P. 11425 ) an den Oberbefehlshaber des Heeres. Berlin, 4. Juli 1940, gez. Göring [Abschrift].

${ }^{29}$ Best urteilte über Michel nach dem Krieg: $»$ Der Leiter der Abteilung Wirtschaft Ministerialdirektor Dr. Michel war ein ausgezeichneter Fachbeamter des Reichswirtschaftsministeriums. Er war mit nüchterner Sachlichkeit allein auf seine Fachaufgabe eingestellt und erschien deshalb menschlich etwas unzugänglich. Da ich - trotz beträchtlichen Temperamentunterschieds - diese Sachlichkeit verstand und schätzte, sind wir gut miteinander ausgekommen und haben einander unterstützt, soweit dies bei der Verschiedenheit unserer Aufgaben erforderlich und möglich war«. BA Koblenz, N 1023/1 (unfoliiert): Werner BEST, Erinnerungen aus dem besetzten Frankreich 1940-1942, o.O., o.J. (Kopenhagen 1951?), 61 S., hier S. 13. Es handelt sich um ein unveröffentlichtes Manuskript, das Best wahrscheinlich auf Anfrage des Instituts für Besatzungsfragen Tübingen gegen Ende seiner Haftzeit in Dänemark oder kurz nach seiner Entlassung in die Bundesrepublik angefertigt hat.

${ }^{30}$ UMBREIT, Militärbefehlshaber, S. 25.

${ }^{31}$ Archives nationales, Paris (AN), AJ 40/578, Akte »Geschäftsordnung«: Geschäftsverteilungsplan der Wirtschaftsabteilung im Verwaltungsstab des MBF, Stand vom 1. Mai 1941.

${ }^{32} \mathrm{Vgl}$. die Organisationsübersicht des Generalbevollmächtigten für die Wirtschaft vom September 1939 in: MÜLLER, Mobilisierung, S. 367.

${ }^{33}$ Siehe den in englischer Übersetzung des Nümberger Dokuments EC-21 in Nazi Conspiracy and Aggression, hg. vom United States Government Printing Office, Bd. VII, Washington D.C. 1946, S. 249 veröffentlichten Geschäftsverteilungsplan: OKH/GenQu (I Wi Gr 9/40) an die Referenten und Sachbearbeiter der Gruppe I Wi. Berlin, 8. Januar 1940, gez. Dr. Posse [Abschrift], ferner BA-MA, RW 19/1769 [unfoliiert]: Rundschreiben des OKW (WFA/Abt. L (IV) Nr. 999/40 g.K.) an die obersten Reichsbehörden betr. Verwaltung der besetzten Gebiete West. Berlin, 10. Mai 1940, gez. Keitel, 4 S., hier S. 2. Allgemein zu den Planungen: UMBREIT, Militärbefehlshaber, S. 4. 
Die Realität der NS-Polykratie im besetzten Frankreich: Doppelarbeit, Kompetenzgerangel und Rivalitäten zwischen den Wirtschaftsdienststellen

Entsprechend der kaum praktikablen Unterscheidung zwischen "ziviler" Kriegswirtschaft und sogenannter Rüstungswirtschaft im äußerst komplizierten institutionellen Gefüge des NS-Staatsapparates, in dem gleich vier oberste Reichsbehörden (OKW, Vierjahresplan, Reichswirtschaftsministerium und Reichsministerium für Bewaffnung und Munition) in ihren kriegswirtschaftlichen Weisungsbefugnissen offen miteinander konkurrierten, bestand auch beim deutschen Besatzungsregime in Frankreich eine vom Reichswirtschaftsministerium und der Wirtschaftsabteilung des Militärbefehlshabers völlig unabhängige Rüstungsorganisation des OKW und der drei Wehrmachtteile. Diese Parallelverwaltung wurde später weitgehend vom Reichsministerium für Bewaffnung und Munition bzw. für Rüstung und Kriegsproduktion übernommen und ausgebaut. Hinzu kam die gleich zu Beginn der Okkupation in Wiesbaden errichtete Deutsche Waffenstillstandsdelegation für Wirtschaft, deren Hauptziel es war, auf dem Verhandlungswege auch die Wirtschaft des unbesetzten Gebiets einschließlich der Kolonien den deutschen Interessen zu unterwerfen. Auch die ständigen Interventionen der Berliner Zentralbehörden in Frankreich erschwerten das Funktionieren der Militärverwaltung zusätzlich. In der Praxis blieb die Aufsicht über die französische Wirtschaft und die Verhandlungen mit Vichy über die immer schwerer auf der Wirtschaftskraft des Landes lastenden Lieferkontingente für Deutschland mit der Ernennung Michels zum Devisen- und AuBenhandelskommissar schon seit $1941^{34}$, im Zuge der territorialen Erweiterung der Machtbefugnisse des Militärbefehlshabers nach der Totalbesetzung Frankreichs aber spätestens seit 1943 der Wirtschaftsabteilung des Majestic vorbehalten. Die Waffenstillstandsdelegation wurde somit mangels eigener Exekutivgewalt immer mehr aus diesem zentralen okkupationspolitischen Aufgabenkreis verdrängt. Hingegen wußte sich der weitverzweigte Apparat der Rüstungsdienststellen, der sich auf ganz Frankreich erstreckte und unter der Ägide Albert Speers seit 1943 zu einem schlagkräftigen Organ der Wirtschaftssteuerung in Frankreich ausgebaut wurde, neben der geballten Fachkompetenz der Technokraten des Majestic zu behaupten. Angesichts der unvermeidlichen negativen Auswirkungen dieser dualen Struktur auf die Effizienz der deutschen Wirtschaftsverwaltung in Frankreich und der sich Dritten bietenden Möglichkeit, die

\footnotetext{
${ }^{34}$ Zentrum für die Aufbewahrung historisch-dokumentarischer Sammlungen, Moskau (ehem. "Sonderarchiv"), Rep. 1458, Findbuch 5, Nr. 7, fol. 3-6: Undatierte Aufzeichnung ohne Unterschrift des Chefs der Militärverwaltung des MBF, 3 S. Anlage I zum Schreiben desselben an den Staatssekretär des RWM. Paris, 26. März 1944, gez. Michel. Siehe auch BA Berlin, R 31.01/2293, fol. 27f.: Ernennungsschreiben des Leiters der Handelspolitischen Abteilung des Auswärtigen Amtes (Ha Pol Frie 652/41) für den Leiter der Wirtschaftsabteilung des MBF. Berlin, 21. Mai 1941, gez. Wiehl.
} 
eine Dienststelle gegen die andere auszuspielen, erwogen die beteiligten Berliner Ressorts Anfang 1944 nach dem Vorbild des NS-Besatzungsregimes in Italien eine Vereinheitlichung der deutschen Wirtschaftsorganisation, die wegen der drohenden alliierten Invasion aber nicht mehr durchgeführt wurde ${ }^{35}$.

\section{Vichy: ein technokratisches Regime?}

Der spektakuläre Aufstieg der Technokraten ${ }^{36}$ in die Schlüsselpositionen des État français ist schon seit längerem von der internationalen Forschung herausgearbeitet $^{37}$. Bereits in der zeitgenössischen Perzeption wurde er als ein Charakteristikum des Regimes erkannt, wenn auch von seinen traditionalistischen wie kollaborationistischen Gegnem eilig zum Komplott der "Synarchie« ${ }^{38}$ verklärt. Zwar ist die Verschwörung einer als "Synarchie« titulierten Geheimorganisation von Technokraten zur Machtübernahme in Vichy längst als politischer Mythos enttarnt ${ }^{39}$, doch hat diese Legende der Forschung lange Zeit den Blick auf die regimeübergreifenden

${ }^{35}$ BA Berlin, R 3/1657, fol. 44: Der Chef des Planungsamtes des Generalbevollmächtigten für Rüstungsaufgaben im Vierjahresplan (Pla 07302/29.4.) an den Leiter des Rüstungsamts im Reichsministerium für Rüstung und Kriegsproduktion. Berlin, 29. April 1944, gez. Hans Kehrl.

${ }^{36} \mathrm{Da}$ der Ausdruck damals noch nicht geläufig war, wurde im Französischen anstelle von "technocrate« häufig das Wort »technicien« gebraucht.

${ }^{37}$ Siehe unter einer Vielzahl von Beiträgen vor allem Robert O. PAXTON, La France de Vichy 1940-1944, Paris 1973, S. 204-213; Richard F. KUISEL, Technocrats and Public Economic Policy: From the Third to the Fourth Republic, in: Journal of European Economic History 2 (1973) S. 53-99; DeRS., Capitalism and the State in Modern France. Renovation and Economic Management in the Twentieth Century, Cambridge, London u.a. 1981, S. 128-131; Philippe BAUCHARD, Les technocrates et le pouvoir. X-Crise, Synarchie, CGT, Clubs, Paris 1966; BRUN, Technocrates, passim; Michèle COINTET-LABROUSSE, Vichy et le fascisme. Les hommes, les structures et les pouvoirs, Brüssel 1987 (Questions au XX $X^{\mathfrak{e}}$ siècle, 2), S. 161-165. Zusammenfassung des aktuellen Diskussionsstandes bei Marc Olivier BARUCH, Servir l'État français. L'administration en France de 1940 à 1944, Paris 1997 (Pour une histoire du XX $\mathrm{XX}^{\mathrm{e}}$ siècle), S. 174-176 u. 220-223.

${ }^{38}$ Der Terminus »Synarchie« umschreibt in seiner geläufigen Bedeutung die Ausübung politischer Herrschaft durch eine homogene Elite zumeist technizistischer Herkunft. Siehe Henry ROUSSO, La collaboration. Les noms, les thèmes, les lieux, Paris 1987, S. 166f.

${ }^{39}$ Zur verschwörungstheoretischen Genesis der "Synarchie « Vichys liegen inzwischen mehrere wissenschaftliche Untersuchungen vor. Siehe vor allem Richard F. KUISEL, The Legend of the Vichy Synarchy, in: French Historical Studies 6 (1970) S. 365-398, femer neuerdings Olivier DARD, La synarchie ou le mythe du complot permanent, Paris 1998 (Terre d'histoire, 2), S. 81-91. Limore YAGIL, La synarchie ou le mouvement "synarchie d'Empire« et Vichy 1940-1944, in: Guerres mondiales et conflits contemporains Nr. 165 (Januar 1992) S. 71-89 und Annie LACROIX-RIZ, Industriels et banquiers français sous l'Occupation. La collaboration économique avec le Reich et Vichy, Paris 1999 (références, histoire contemporaine, 21), S. 19-26, verteidigen hingegen die These vom "Komplott der Synarchie«. 
Kontinuitäten verstellt. Ein großer Teil der wirtschafts- und sozialpolitischen "Nonkonformisten« der 1930er Jahre findet sich unter den Technokraten Vichys (und der IV. wie der V. Republik) wieder ${ }^{40}$. Sie verband neben ihrer gleichen sozialen Herkunft die Sozialisation als Absolventen der Grandes Écoles (Normale, Polytechnique, Sciences po). Ihr Aufstieg an die Staatsspitze erfolgte nicht über Nacht nach der Geburt des neuen Regimes, sondern bildete den vorläufigen Höhepunkt einer langjährigen Karriere, die durch ihre Verwaltungstätigkeit in der III. Republik geprägt war ${ }^{41}$, deren politischen Grundwerten sie aber häufig in technizistischer Indifferenz gegenüberstanden. Trotz aller teleologischen Gegensätze in der Frage der Umsetzung der allseits postulierten Staatsreform war ihr Antiparlamentarismus nicht weniger ausgeprägt als derjenige der Traditionalisten des Regimes: "Les technocrates d'allégeance ou simplement de tempérament se sont de suite sentis attirés par les possibilités que Vichy semblait leur offrir. [...] L'occasion leur paraît tentante d'une réforme totale de l'État, soustrait à l'incompétence parlementaire, remis enfin entre les mains des spécialistes ${ }^{42}$. Der ebenso plötzliche wie unerwartete Wegfall der verhaßten parlamentarischen Kontrolle ließ fur viele angesichts der Versuchung, endlich die lang ersehnte innenpolitische Tabula rasa (»Révolution nationale «) in die Tat umsetzen zu können, die neuen politischen Restriktionen infolge der Präsenz der Besatzer in den Hintergrund treten. Planungswütige Ungeduld und antidemokratische Geistesverwandtschaft mit den Kollegen der deutschen Besatzungsstäbe hingen mit der Kollaborationswilligkeit vieler Technokraten des Regimes unmittelbar zusammen, sie können daher auch als der eigentliche Motor der Kollaborationspolitik definiert werden.

\section{Die ökonomische Globalsteuerung als Dreh- und Angelpunkt der technokratischen Reorganisation des französischen Staatsapparates}

Die neuen Institutionen der »économie dirigée« reflektieren sowohl nach innen den endgültigen Durchbruch staatsinterventionistischer Wirtschaftspolitik in

\footnotetext{
${ }^{40}$ Stanley HofFMANN, Collaborationism in France during World War II, in: Journal of Modern History 40 (1968) S. 389. Zur Kontinuitätsthese siehe ferner Robert O. PAXTON, Vichy France. Old Guard and New Order 1940-1944, New York 1972, S. 352-357 sowie Bertram M. GORDON, Collaborationism in France during the Second World War, Ithaca und London 1980, S. 24. Roger BOURDERON, Le régime de Vichy était-il fasciste? Essai d'approche de la question, in: Revue d'histoire de la Deuxième Guerre mondiale Nr. 91 (Juli 1973) S. 42 spricht in diesem Zusammenhang vom "prätechnokratischen « Charakter des Regimes. 4! Jean-Louis LOUBET DEL BAYLE, Les non-conformistes des années 30. Une tentative de renouvellement de la pensée politique française, $\operatorname{Paris}^{2} 2001$ (XX ${ }^{e}$ siècle).

${ }^{42}$ André SIEGFRIED, Le Vichy de Pétain, le Vichy de Laval, in: Revue française de science politique 6(1956) S. $743 \mathrm{f}$.
} 
Frankreich, als auch nach außen die strategische Option Vichys zugunsten der Staatskollaboration, indem dem NS-Besatzungsapparat in Frankreich ein systematisch ausgebauter und mit geballter Sachkompetenz unterfütterter Verwaltungsapparat gegenübergestellt wurde. Das ökonomische Krisenmanagement des Regimes war aber keineswegs nur für den Augenblick gedacht. Die materiellen und politischen Zwänge, die sich aus den Kriegsereignissen und der Anwesenheit der Besatzer ergaben, waren gewiß eher kurzfristiger Natur, aber die klare Abkehr von der wirtschaftspolitischen Tradition des Laissez faire beruhte auf Reformprojekten, die eindeutig auf Dauer angelegt waren ${ }^{43}$.

Der Aufbau der neuen Organisation erfolgte in mehreren Etappen, ohne jemals vollendet $\mathrm{zu}$ sein. Hierbei wich die administrative Praxis wegen der laufenden Anpassungen an die zunehmenden inneren und äußeren Zwänge zum Teil deutlich von den idealtypischen Mustern ab, wie sie sich auf dem Papier in den anspruchsvollen Organisationsschemata der planungswütigen Technokraten Vichys niederschlugen. Dennoch konstituierten sich die Hauptorgane des Lenkungsapparates bereits in den ersten Wochen des Regimes: Neben dem Ministerium für Industrieproduktion (ministère de la Production industrielle) entstanden zwei völlig neue Institutionen, die diesem direkt unterstellt waren: die Organisationsausschüsse (comités d'organisation) und das Zentralbewirtschaftungsamt für Industrieerzeugnisse (Office central de répartition des produits industriels). Vor allem das grundlegend reorganisierte Industrieministerium, das unter Vichy - im krassen Gegensatz zu seinem Schattendasein während der III. Republik - im Zuge der Integration benachbarter Wirtschaftsressorts zum Superministerium avancierte, entwickelte sich zum administrativen Mittelpunkt des Interventionsstaates, eine Funktion, die es auch in der Nachkriegszeit behielt. Auch seine interne Organisationsstruktur blieb trotz veränderter Aufgaben praktisch bis heute unverändert ${ }^{44}$. Mit seinem umfangreichen Apparat zentraler Warenrationierung und halbstaatlicher Wirtschaftsverbände wurde die Tausende von Mitarbeitern zählende Mammutbehörde ${ }^{45}$ zum Inbegriff der Technokratie Vichys.

\footnotetext{
${ }^{43}$ MargairaZ, État, Bd. I, S. 511.

${ }^{44}$ Henry ROUSSO, Le ministère de l'Industrie dans le processus de planification: une adaption difficile (1940-1969), in: DERS. (Hg.), De Monnet à Massé. Enjeux politiques et objectifs économiques dans le cadre des quatre premiers plans (1946-1965), Paris 1986, S. 27-40, hier S. 27.

${ }^{45}$ Nach einer internen Statistik belief sich die Gesamtzahl der Angehörigen des Ministeriums im September 1944 - also unmittelbar nach dem Ende des Regimes - auf 29937 Mitarbeiter, darunter allein 8800 im Zentralbewirtschaftungsamt und 13200 in den Organisationsausschüssen, unter ihnen waren 6537 Beamte. Allein diese Zahlen veranschaulichen die reale Dimension des Lenkungsapparates. Siehe Henry Rousso, Les élites économiques dans les années quarante, in: Mélanges de l'École française de Rome, Abt. Moyen âge/Temps modernes 95 (1983), Teil 2, S. 29-49, hier S. 49.
} 


\section{Das Kabinett Darlan: politischer Durchbruch der technokratischen Elite}

Die entscheidende Rolle spielte hierbei eine Riege qualifizierter Nachwuchskräfte, die hervorragende administrative Sachkenntnisse mit Karriereerfahrungen in Großindustrie und Hochfinanz verbanden. Diese neue technokratische Elite besa $\beta$ zum Teil bereits leitende Positionen in der Ministerialbürokratie und stieg Anfang 1941 mit der Konstituierung des neuen Kabinetts unter Admiral François Darlan in die Spitze des Staatsapparates vor. Personalisiert wurde dieser Prozeß durch den spektakulären Aufstieg der »équipe Worms«, einem kleiner Kreis junger Technokraten um Gabriel Le Roy Ladurie, Generaldirektor der Banque Worms, die Ende Februar 1941 gemeinsam ins französische Kabinett eintraten und seitdem unmittelbar politische Herrschaftsbefugnisse ausübten. $\mathrm{Zu}$ ihnen zählten u.a. Pierre Pucheu, Jacques Barnaud und François Lehideux, Jacques Benoist-Méchin und Paul Marion; die beiden letzteren hatten sich allerdings nicht als Wirtschaftsmanager, sondern als Schriftsteller bzw. politische Publizisten einen Namen gemacht und galten als »langjährige Freunde « von Otto Abetz, dem deutschen Botschafter in Paris ${ }^{46}$. Pucheu übernahm das Industrieressort, Barnaud wurde zum Generaldelegierten für die deutsch-französischen Wirtschaftsbeziehungen ernannt, Lehideux fungierte als Generalbeauftragter für nationale Wirtschaftsplanung, BenoistMéchin und Marion avancierten zu beigeordneten Generalsekretären beim stellvertretenden Ministerpräsidenten und faktischen Kabinettschef Darlan. Sie kamen zwar ursprünglich aus völlig entgegengesetzten politischen Milieus, vertraten aber inzwischen dieselben Grundüberzeugungen: die Notwendigkeit einer »Revolution « von oben durch einen engen Personenkreis mit maximalen intellektuellen und "technischen « Fähigkeiten zur »Modernisierung « und »Rationalisierung" der wirtschaftlichen und administrativen Strukturen Frankreichs unter einer jeder parlamentarisch-demokratischen Hürden entledigten elitären Diktatur von $»$ Technikern ${ }^{47}$. Diese Auffassung war im wahrsten Sinne des Wortes totalitär ${ }^{48}$ : Völlig losgelöst von einer pluralistischen Interessenartikulation sollte das Land fortan ausschließlich nach den Gesichtspunkten

${ }^{46}$ Politisches Archiv des Auswärtigen Amts, Berlin (PA-AA), R 106149, fol. 313833: Geheimes Fernschreiben (ohne Nummer) der Deutschen Botschaft Paris an das Auswärtig Amt (AA), Paris, 18. April 1942, 14.20 Uhr. Zur Haltung der französischen Intellektuellen gegenüber der NS-Propaganda der europäischen »Neuordnung« siehe jetzt vor allem Bernard BRUNETEAU, »L'Europe nouvelle« de Hitler. Une illusion des intellectueis de la France de Vichy, Monaco 2003 (Démocratie ou totalitarisme) sowie Albrecht BETZ, Stefan MARTENS, (Hg.), Les intellectuels et l'Occupation, 1940-1944. Collaborer, partir, résister, Paris 2004.

${ }_{47}$ Jacques BENOIST-MÉCHIN, De la défaite au désastre, Bd. 1: Les occasions manquées, juillet 1940-avril 1942, Paris 1984, S. 54 u. 57.

${ }_{48}$ Henry Rousso, Les paradoxes de Vichy et de l'Occupation. Contraintes, archaïsmes et modernités, in: Patrick FrIDENSON, André STRAuS ( $\mathrm{Hg}$.), Le capitalisme français, $\mathrm{XIX}^{\mathrm{e}}-\mathrm{XX}^{e}$ siècle. Blocages et dynamismes d'une croissance, Paris 1987, S. 79. 
technischer Effizienz regiert werden. Dem entsprach ihre radikale Ablehnung des politischen Systems der III. Republik wie der liberalen Demokratie überhaupt, desgleichen die partielle ideologische Affinität zum Nationalsozialismus, die sich besonders in ihrer Faszination gegenüber der Wirtschaftsorganisation und ökonomischen Expansion des "Dritten Reiches« sowie der offiziellen Überwindung des Klassenantagonismus durch die scheinbare soziale Harmonie in »Hitlers Volksstaat ${ }^{49}$ manifestierte ${ }^{50}$.

Trotz des politischen Gewichts der Technokraten, insbesondere auf dem Sektor der Wirtschaftspolitik, wäre es verkürzt, das aus der Niederlage von 1940 hervorgegangene Regime als durchgehend technokratisch zu definieren. Die jungen »Techniker« bzw. "Technokraten« innerhalb des Kabinetts symbolisieren eine kollaborationistische Strömung des Regimes, die sich mit ihrer Fokussierung auf den technischen Fortschritt und ihren illusionären Hoffnungen auf den industriellen Aufbruch Frankreichs im »Neuen Europa» der Nationalsozialisten ${ }^{51}$ in diametralem Gegensatz zur offiziellen Doktrin des »retour à la terre« befand. Gerade diese Janusköpfigkeit zwischen »Modernisten« einerseits und »Traditionalisten« andererseits, die im Kabinett Darlan besonders hervorstach, stellt ein signifikantes Strukturmerkmal der Vichy-Administration dar. Sie veranlaßte den »traditionalistischen « Staatssekretär Henri Moysset zu der bekannten Äußerung gegenüber Darlan, die den »Modernisten« des Regimes ihren Namen gab: "Votre ministère me rappelle le nom d'un café de ma ville natale du Ségalas: aux jeunes cyclistes et aux anciens Romains « ${ }^{52}$.

Die Bedeutung ideologischer und ökonomisch-pragmatischer Faktoren in der Kollaborationspolitik der »jeunes cyclistes«: der Fall Bichelonne

Trotz der Affinitäten der »jeunes cyclistes « zum Nationalsozialismus basierte namentlich bei Barnaud, Pucheu und Lehideux das Ziel einer möglichst engen - sowohl ökonomischen als auch politisch-militärischen - Zusammenarbeit mit der Okkupationsmacht weniger auf ideologischem Affekt als auf wirtschaftsrationaler und geostrategischer Reflektion ${ }^{53}$, so daß ihre Kollaborati-

${ }^{49}$ So der provokative Titel der umstrittenen Neuerscheinung von Götz ALY, Hitlers Volksstaat. Raub, Rassenkrieg und nationaler Sozialismus, Frankfurt a. M. 2005.

${ }^{50}$ KUISEL, Technocrats, S. 79.

"II Ibid; ferner Betram M. GORDON, The morphology of the collaborator: the French case, in: Journal of European Studies 23 (1993) S. 1-25, bes. S. 11-13.

${ }_{52}$ Zit. n. Robert ARON, Histoire de Vichy 1940-1944, Paris 1954, S. 378.

${ }^{53}$ Hierunter fiel vor allem ihr Ziel, Frankreich im Rahmen der NS-»Neuordnung « zum atlantischen Brückenkopf Europas zu machen. Archives du ministère des Affaires étrangères, Paris (MAE), Papiers 1940, Abetz, Bd.2, fol. 193-202: "Plan einer Neuordnung Frank- 
onspolitik im Unterschied zu den Pariser Ultrakollaborationisten um Marcel Déat und Jacques Doriot eher Ausdruck pragmatischer Kalkulation als wirklich faschistischer Überzeugungen war $^{54}$. Die primär opportunistische Motivation ihres Handelns blieb auch den Besatzungsbehörden nicht verborgen: Abetz meldete an das Auswärtige Amt, die Gruppe um Barnaud, Pucheu und Lehideux "verfolgt heute aus wirtschaftlichen Nützlichkeitserwägungen die Politik eines immer stärkeren Einbaus der französischen Industrie und des französischen Handels in das von Deutschland geführte Europa ${ }^{55}$.

Zum Prototyp schlechthin der jungen Technokraten des Regimes avancierte aber nicht ein Mitglied der »équipe Worms«, sondern Jean Bichelonne, der von April 1942 bis zur Befreiung Frankreichs an der Spitze des Industrieministeriums stand. Ihm eilte seit langem der Ruf eines Wirtschaftsexperten mit enzyklopädischem Wissen und einzigartiger Fachkompetenz voraus, so daß er bereits in seiner früheren Funktion als Hauptabteilungsleiter (secrétaire général à l'Industrie et au Commerce intérieur) nicht nur innerhalb der VichyAdministration, sondern auch unter den deutschen Wirtschaftsdienststellen als »kommender Mann ${ }^{56}{ }^{6}$ des Regimes gehandelt wurde. Der Fall Bichelonne reflektiert nahezu idealtypisch die signifikante Ambivalenz der »jeunes cyclistes«, bei denen brillante Intelligenz gepaart mit politischer Radikalität eine singuläre Symbiose einging. Die in der Memoirenliteratur der Vichy-Akteure kolportierte These über Bichelonne als technisch genialer, aber politisch naiver Wirtschaftsexperte wurde von der Forschung kaum kritisch hinterfragt. Insofern hat das geläufige Erklärungsmuster des sogenannten KwaïSyndroms $^{57}$, der Minister für Industrieproduktion und Hauptorganisator der

reichs« (Aufzeichnung ohne Unterschrift), o.D. [Anfang April 1941], deutsche Übersetzung des französischen Originals (Anlage zum Schriftbericht des Deutschen Botschafters Paris an das AA vom 4. April 1941), $10 \mathrm{~S}$., hier fol. 195, ferner als französische Rückübersetzung veröffentlicht in Otto ABETZ, Pétain et les Allemands. Memorandum d'Abetz sur les rapports franco-allemands, Paris 1948, S. 79-84, hier S. 82.

${ }^{54}$ RouSSO, Collaboration, S. 167; Yves DURAND, Le nouvel ordre européen nazi. La collaboration dans l'Europe allemande (1938-1945), Brüssel 1990 (Questions au XX $X^{\mathfrak{e}}$ siècle, 20) S. 239; Jean-Pierre AZÉMA, Vichy, l'héritage maudit, in: L'Histoire Nr. 162 (Januar 1993) S. 103.

55 MAE, Papiers 1940, Abetz, Bd. 1, fol. 82-88: Undatierter Schriftbericht der Deutschen Botschaft Paris an das AA für den Monat April 1941, gez. Abetz, hier B1. 86 (Hervorhebung vom Vf.).

${ }^{56}$ AN, AJ 40/540, Akte »Generaldelegierter der franz. Regierung«, Bd. I (Juli 1940-Februar 1942): Anonyme Aufzeichnung des MBF/Verwaltungsstab/Abt. Verwaltung/Gruppe 2 (Polizei) für Kriegsverwaltungschef Best. Paris, 21. Februar 1942, S. 6.

${ }^{57}$ Alfed Sauvy, De Paul Reynaud à Charles de Gaulle. Un économiste face aux hommes politiques 1934-1967. Scènes, tableaux et souvenirs, Tournai 1972, S. 134: "Pourquoi cet homme est-il passé à la collaboration? C'est l'histoire du pont de la rivière Kwaï; il a été emporté par la technicité et le désir de mettre les choses en ordre«; Ibid. S. 142: »Cet homme est victime de la maladie de l'organisation, de l'ordre, de la technicité «, Wiederaufnahme der These in: DERS., La vie économique des Français de 1939 à 1945, Paris 1978, S. 177. 
ökonomischen Kollaboration mit NS-Deutschland sei sich über den politischen Kontext und die weitreichenden Konsequenzen seines Handelns gar nicht bewußt gewesen, auch in verdienstvolle wissenschaftliche Untersuchungen Eingang gefunden ${ }^{58}$. Dem Mythos vom gutgläubigen Mustertechnokraten Vichys und vom richtigen Mann zur falschen Zeit ${ }^{59}$ widersprechen zeitgenössische französische und deutsche Quellen. In ihnen zeigt sich ein anderer Bichelonne, der durchaus eine erkennbare politische Identität besa $\beta^{60}$.

Tatsache ist, daß Bichelonne nicht zu den opportunistischen Technokraten zählte, die dem Regime nach der Kriegswende im Winter 1942/1943 den Rükken kehrten, sondern er blieb bis zum Ende des Regimes auf seinem exponierten Posten. Was auf der einen Seite als eklatanter Realitätsverlust, ja politische Naivität des Ministers für Industrieproduktion erscheint, kann auf der anderen Seite aber auch als wohldurchdachte strategische Option und konsequentes Festhalten an der Illusion der partnerschaftlichen Integration Frankreichs in die nationalsozialistische "Neuordnung " Europas gedeutet werden, zu der er aufgrund seiner antikommunistischen und antidemokratischen Grundüberzeugungen keine Alternative sah. Bereits Milward erkannte, daß Bichelonne trotz der sich häufenden militärischen deutschen Rückschläge auch noch 1943/1944 ganz bewußt den eingeschlagenen Weg der Kollaboration mit den NSBesatzungsorganen fortsetzte im festen Glauben, die Rettung Frankreichs wäre nur in Zusammenarbeit mit Deutschland und "Europa« möglich ${ }^{61}$. Seine unerschütterliche Loyalität gegenüber der Okkupationsmacht wurde durch die exklusive Möglichkeit ostentativ honoriert, auch in der deutschen Presse "die neuen französischen Einrichtungen«, die insbesondere »für die Errichtung einer wahren europäischen Wirtschaft sehr wertvoll ${ }^{62}$ seien, in extenso darzulegen. Das innenpolitische Pendant zur prodeutschen Haltung bestand aus einer klaren Absage an den Parlamentarismus. Allein in einem autoritären Staat war in seinen Augen eine wintelligente ${ }^{63}$, von der öffentlichen Hand aktiv nach einheitlichen Parametern gesteuerte Wirtschaft durchsetzbar.

${ }^{58}$ MARGaIRAZ, État, Bd. I, S. 678; BARUCH, Servir, S. 370.

${ }^{59}$ Rousso, Collaboration, S. 14: "Sans les circonstances tragiques de l'Occupation, Bichelonne aurait sans doute fait partie des >modernisateursı de l'après-guerre«.

${ }^{60}$ BA-MA, RW 35/244, fol. 48: "Allgemeiner Rückblick « (wie Anm. 25)

${ }^{6}$ Alan S. MiLWARD, The New Order and the French Economy, Oxford 1970, S. 175.

62 Jean BICHELONNE, Französische Wirtschaftspolitik im Rahmen des neuen Europa, in: Die Deutsche Volkswirtschaft 11 (1942) S. 1391-1395 (Themenschwerpunkt: wKonstituierung der europäischen Wirtschaftsgemeinschaft. Teil III: Das Bewußtsein der Zusammengehörigkeit«).

63. $\mathrm{Zu}$ seiner wirtschaftspolitischen Konzeption einer méconomie logique, intelligente et humaine « siehe AN, 72 AJ/1926: Extraits des conférences prononcées par M. Bichelonne, Ministre Secrétaire d'État à la Production Industrielle et aux Communications à l'Hôtel de Ville de Paris les 5 août et 12 octobre 1943. Druck, o.O., o.J. [Paris, 1943], 24 S., bes. S. 1 u. 14 (Vortrag vom 5. August 1943). 
Zur Realisierung seiner ideologischen Ziele fehlte es dem Mustertechnokraten des Regimes nicht an elitärem Selbstbewußtsein und politischem Ehrgeiz. Ihm wurden im März 1943 sogar Ambitionen auf den Posten des Regierungschefs nachgesagt ${ }^{64}$. Seine Politisierung korrelierte mit seiner exponierten Stellung als de facto zweitwichtigstes Kabinettsmitglied hinter Laval selbst ${ }^{65}$ und ging über den »unpolitischen « Habitus des Technokraten weit hinaus. Sie wird in einem Sonderbericht der Deutschen Botschaft anläßlich seiner Reise ins Führerhauptquartier "Wolfsschanze« am 18. Dezember 1942 deutlich, als er als einziger Vichy-Minister Laval zu seiner Unterredung mit Hitler begleitete: „Bichelonne ist unzweifelhaft einer der fähigsten französischen Minister. Die Botschaft konnte mehrfach feststellen, daß er die Frankreich gestellten Probleme in aller Klarheit erkennt und hierbei eine großzügige Betrachtungsweise an den Tag legt. Er steht aufrichtig hinter der Politik Lavals, die er energisch vertritt ${ }^{66}$. Diese Beurteilung Bichelonnes findet in einem eingehenden Drahtbericht der Botschaft vom Juli 1943 noch einmal eine ausdrückliche Bestätigung: "Die Regierung steht so praktisch auf den beiden Augen Lavals, neben seinem alten Vertrauten, Finanzminister Cathala und dem fachlich und organisatorisch hoch befähigten Produktionsminister Bichelonne, auf deren Ergebenheit er völlig vertrauen kann, sind die übrigen Minister keine politischen Persönlichkeiten, sondern mehr Beamte ${ }^{67}$. Gewiß zählte Bichelonne nicht zu den markanten politischen Figuren des Regimes wie Pétain oder Laval. Die pauschale Klassifizierung als apolitischer Wirtschafts- und Verwaltungsfachmann, der aufgrund der widrigen Zeitumstände einem tragischen Mißverständnis erlag, widerspricht aber nicht nur seinem (politischen) Selbstverständnis, sondern auch den historischen Tatsachen.

\section{Ökonomische Interessenkonvergenzen und gegenseitige Beeinflussung des Vichy- und NS-Regimes}

Die Kollaboration der technokratischen Funktionseliten Vichys mit denen der NS-Okkupationsmacht war bedingt durch partielle Interessenkonvergenzen. Hierzu zählt vor allem das gemeinsame Ziel der Errichtung staatsinterventionistischer Strukturen in Frankreich, in dem sich sehr unterschiedliche, teilweise konträre Motive trafen (ökonomisches Krisenmanagement und Wirtschaftsre-

\footnotetext{
${ }^{64}$ Pierre NiCOLLE, Cinquante mois d'Armistice. Vichy, 2 juillet 1940-26 août 1944. Journal d'un témoin, Paris 1947, Bd. II, S. 126 (Notiz vom 11. März 1943).

${ }^{65}$ Pétain wurde sukzessiv auf die repräsentativen Aufgaben des Staatschefs zurückgedrängt.

${ }^{66}$ PA-AA, R 29597, fol. 118455f.: Fernschreiben der Deutschen Botschaft Paris (Nr. 6015) an das AA Berlin. Paris, 17. Dezember 1942, gez. Schleier (Hervorhebung vom Vf.).

${ }^{67}$ Geheimes Fernschreiben der Deutschen Botschaft Paris (Nr. 5024) an das AA. Paris, 27. Juli 1943, gez. Schleier, in: ADAP, Serie E, Bd. VI, S. 308-324 (Dok. 182), hier S. 313.
} 
form nach der Niederlage im Westfeldzug einerseits, Rationalisierung der kriegswirtschaftlichen Ausbeutung andererseits). Ein Paradebeispiel hierfür war die schnelle Einführung staatlicher Warenbewirtschaftung, die aufgrund der Bemühungen Vichys, die administrative und ökonomische Einheit des Landes zu erhalten, gleichzeitig im besetzten wie im unbesetzten Gebiet eingeführt wurde. Sie verhinderte zwar den Zusammenbruch der Versorgung der französischen Bevölkerung, bildete aber den Grundstein für die rationelle Ausbeutung der Wirtschaft auch der unbesetzten Zone zugunsten der NS-Kriegführung, indem Betriebe, die für deutsche Auftraggeber arbeiteten, in der Rohstoff- und Energieversorgung bevorzugt wurden. Trotz der im Waffenstillstand vertraglich zugesicherten französischen Souveränität gehörte damit das einer engen deutschen Kontrolle unterworfene Zentralbewirtschaftungsamt in Paris zu den wichtigsten Ausbeutungsinstrumenten der Besatzungsmacht und entwickelte sich zu einem Hauptorgan der kriegswirtschaftlichen Integration Frankreichs.

Diese Interessenkonvergenzen bilden gleichzeitig einen wesentlichen Faktor nicht nur der täglichen Interaktion, sondern auch der längerfristigen gegenseitigen Beeinflussung beider Regime, insbesondere in der Wirtschaftspolitik. Sowohl bei der Einfuihrung zentraler Warenbewirtschaftung als auch bei der Errichtung wirtschaftlicher Zwangsverbände in Form der Organisationsausschüsse ist der bekannte Einfluß des deutschen Modells (Reichsstellen und Wirtschaftsbzw. Fachgruppen) nicht von der Hand zu weisen. Weitaus weniger geläufig ist allerdings die Feststellung, daß auch die zentralen Lenkungsbehörden in Berlin sich an den Erfahrungen auf dem französischen Experimentierfeld orientierten. Schon bei der Errichtung des Zentralbewirtschaftungsamtes und der Organisationsausschüsse wurden von vornherein die administrativen Fehler vermieden, die in den ersten Kriegsjahren zu beträchtlichen Effizienzverlusten in der deutschen Kriegswirtschaft führten. Namentlich das Pariser Zentralbewirtschaftungsamt, das im Gegensatz zu den Organisationsausschüssen unter maßgeblicher deutscher Beteiligung entstand, kann - der üblichen Rivalität zwischen den Berliner Ressorts entledigt - als deutsches Modell der Wirtschaftslenkung in Reinkultur bezeichnet werden ${ }^{68}$. Die Speer-Administration profitierte anläßlich der überfälligen Reorganisation der deutschen Kriegswirtschaft 1942/43, insbesondere bei der Errichtung der Lenkungsbereiche und des Planungsamtes, von den Erfahrungen in Frankreich. Auch das Beispiel der französischen Organisationsausschüsse mit ihren für deutsche Verhältnisse bis dahin unerhörten hoheitlichen Aufgaben ${ }^{69}$ stand bei der Errichtung der deutschen Ausschüsse und Rin-

${ }^{68}$ BA Berlin, R 4603/1948, fol. 2-15: Planungsamt/Generalreferent für Sonderaufgaben $(\mathrm{D} / \mathrm{n})$ : Vermerk über den Besuch des Office central in Paris am 13./14.3.44 durch Dr. Dom, Dr. Wagenfuihr und Dr. Elmendorff. Berlin, 24. März 1944, hier fol. 2.

${ }^{69}$ Der umfassende Aufgabenbereich der Organisationsausschüsse führte daher anfangs zu schweren Konflikten mit den Besatzungsdienststellen. Siehe Ame RADTKE-DELACOR, La position des comités d'organisation face aux autorités d'occupation: La pomme de discorde 
ge Pate. Desgleichen manifestiert sich der Einfluß des französischen Beispiels eines Superministeriums für Industrieproduktion bei der Entstehung des Reichsministeriums für Rüstung und Kriegsproduktion keineswegs nur in der terminologischen Analogie beider Ressorts. Das Interesse der Berliner Zentralstellen an den französischen Musterschülern im Zentralbewirtschaftungsamt, die ihre deutschen Lehrmeister bald übertrafen, führte 1943/1944 zu einem regelrechten Tourismus deutscher Planungsspezialisten nach Frankreich. Die Technokraten des Berliner Planungsamtes waren beeindruckt, wie es dem Zentralbewirtschaftsungsamt unter der tatkräftigen "Amtshilfe» des deutschen Generalbeauftragten Wilhelm Weniger gelang, "die Gedankengänge der deutschen Bewirtschaftung in vorbildlicher Klarheit auf die französischen Verhältnisse zu übertragen«. Insofern war es für sie »beinah schmerzlich«, feststellen zu müssen, »daß viele Fehler und Unzulänglichkeiten, um deren Bereinigung wir in Deutschland noch bemüht sind, in Frankreich dadurch beseitigt werden konnten, daß Herr Weniger unbeirtt durch die Positionen anderer Persönlichkeiten es ermöglicht hat, seine Gedankengänge in die Praxis umzusetzen ${ }^{70}$.

\section{Die Wirtschaftskollaboration in den Augen der Technokraten des Majestic}

Die Ausgestaltung der Besatzungsbehörden als "Aufsichtsverwaltung " hatte zur Folge, daß der Militärbefehlshaber allein schon aus Personalmangel auf die französische Staatskollaboration zur Wahrnehmung der von Berlin aus übertragenen umfangreichen Aufgaben, vor allem in der Kriegswirtschaft, dringend angewiesen war - eine Tatsache, die den Franzosen nicht verborgen bleiben konnte. Michel benannte in seinen an die Berliner Ressorts adressierten Sonderberichten explizit den eigentlichen Schwachpunkt der deutschen Position: "Schon jetzt aber reichen bei der Wirtschaftsabteilung in Frankreich die vorhandenen Kräfte nicht mehr aus, den ständig steigenden Aufgaben überall so gerecht zu werden, wie es nötig ist bei der Zusammenarbeit mit den Einrichtungen eines bisher feindlichen Staates, dessen Bevölkerung keineswegs überwiegend auf deutscher Seite steht ${ }^{7 !}$. Im Unterschied zur Deutschen Botschaft war

des commandes allemandes en 1940-1941, in: Hervé JOLY (Hg.), Les comités d'organisation et l'économie dirigée du régime de Vichy. Actes du colloque international, Caen, 3./4. April 2003, Caen 2004, S. 63-71.

${ }^{70}$ BA Berlin, R 4603/1948, fol. 2: Vermerk über den Besuch des Office central (wie Anm. 68). Zur amtlichen deutschen Bewirtschaftungsdoktrin siehe Wilhelm WENIGER, Bewirtschaftung der Industrieprodukte in Frankreich, in: Der Vierjahresplan. Zeitschrift für nationalsozialistische Wirtschaftspolitik 6 (1942) S. 366f.

71 BA-MA, RW 35/304, fol. $181 \mathrm{ff}$ : MBF/Verwaltungsstab/Wirtschaftsabteilung: Die Entwicklung und Lage der französischen Wirtschaft im Bereich des Militärbefehlshabers in 
die Perzeption der französischen Kollaborationspolitik durch die Militärverwaltung nicht von politischem Idealismus, sondern ökonomischem Pragmatismus, ja okkupationstaktischem Zynismus geprägt. Unter der Maxime einer optimalen Ausbeutung der französischen Ressourcen erschöpfte sie sich darin, die französischen Souveränitätsrechte mittels der Delegierung eines Maximums unpopulärer Aufgaben an den Staatsapparat Vichys zu instrumentalisieren ${ }^{72}$ : "Dieses System hat nicht nur den Vorteil des sparsamsten Einsatzes deutscher Fachkräfte, sondern durch die Zwischenschaltung französischer Dienststellen wird auch die Verantwortung der französischen Regierung gegenüber dem französischen Volk klar herausgestellt und werden die zu treffenden, oft sehr einschneidenden Maßnahmen auch in der zweckmäßigsten Form an die französische Bevölkerung herangetragen ${ }^{73}$. Die Technokraten des Majestic konnten in einer Zwischenbilanz nach knapp zwei Jahren befriedigt feststellen, daß das okkupationspolitische Kalkül vom Sommer 1940 größtenteils aufgegangen war:

Zusammenfassend ist zu sagen, daß die französische Wirtschaft in großem Umfang für die deutsche Versorgung und die deutsche Fertigung herangezogen ist. Die Umstellung des Produktionsapparates auf kriegswirtschaftliche Zwecke ist weitgehend durchgeführt. Der französische Verbrauch ist auch an Nahrung, Bekleidung, Schuhwerk, Heizung zum Teil erheblich unter den deutschen Verbrauch gesunken und durchweg auf das für die Erhaltung des wirtschaftlichen Lebens des Landes und der Arbeitsfähigkeit der Bevölkerung unentbehrliche Mindestmaß eingeschränkt. Dieses Ergebnis ist unter Mitwirkung der französischen amtlichen Stellen und der Führung der französischen Wirtschaft erzielt worden ${ }^{74}$.

Die Instrumentalisierung der französischen Kollaborationspolitik durch die NS-Besatzungsmacht degradierte Frankreich bereits Anfang 1942 zum unmündigen Lieferanten, dessen Rolle sich lediglich darauf zu beschränken hatte, die wachsenden Forderungen des Reiches zu erfüllen. Göring faßte den deutschen Standpunkt mit dem bekannten Satz zusammen: »Kollaboration der Herren Franzosen sehe ich nur in folgendem: wenn sie abliefern, bis sie selber nicht mehr können, wenn sie es freiwillig tun, dann werde ich sagen, ich kollaboriere ${ }^{75}$. Von den in Vichy erhofften deutschen Gegenleistungen, die nicht

Frankreich im Jahre 1941. Anlage zum Rundschreiben Michels (Wi I 580/42 geh.) an die Berliner Ressorts vom 1. Februar 1942, hier fol. 190v.

${ }^{72}$ Rousso, Collaboration, S. 28; MARGaIRAZ, État, Bd. I, S. 526-530.

${ }^{73}$ Elmar MICHEL, Gelenkte Wirtschaft in Frankreich, in: Berliner Börsen-Zeitung Nr. 166 vom 10. April 1942.

${ }^{74}$ AA/Handelspolitische Abteilung (HaPol. Ila 1186): Aufstellung über die wirtschaftlichen Leistungen Frankreichs zugunsten Deutschlands und die deutschen Lieferungen an Frankreich. Berlin, 19. März 1942. ADAP, Serie E, Bd. II, S. 92 (Dok. 52).

${ }^{75}$ Nürnberger Dok. USSR-170: Geheim. Stenographischer Bericht über die Besprechung des Reichsmarschalls Göring mit den Reichskommissaren für die besetzten Gebiete und den Militärbefehlshabern über die Emährungslage am Donnerstag, dem 6. August 1942, 4 Uhr nachm., im Hermann-Göring-Saal des Reichsluftfahrtministeriums, in: Der Prozeß gegen die Hauptkriegsverbrecher vor dem Internationalen Militärgerichtshof Nürnberg 14. November 
wieder indirekt in die NS-Kriegswirtschaft zurückflossen, war längst keine Rede mehr. In der administrativen Praxis war daher die »Kollaboration« der französischen Wirtschaftstechnokratie nicht wirklich reziprok, sondern entpuppte sich bei genauerer Betrachtung als Einbahnstraße ${ }^{76}$.

\section{Résumé français}

Durant la Seconde Guerre mondiale, l'interventionnisme étatique et le dirigisme économique atteignirent, à la fois en France et en Allemagne, des proportions inconnues jusque-là, dont l'expression manifeste fut la montée en puissance des élites de fonctionnaires technocrates dans les deux États. L'étude des buts, des acteurs, des méthodes et des résultats du dirigisme économique de Vichy et de l'économie de guerre national-socialiste a surtout fait l'objet d'approches historiques nationales. Il manque une analyse systématique et comparative des structures économiques et des élites technocrates des deux pays à cette époque. La présente contribution ne peut pas combler cette lacune, mais tente de soumettre à une analyse comparative les structures technocrates établies en France pendant les quatre années d'interaction entre la puissance d'occupation national-socialiste et le régime de Vichy. Elle met en lumière non seulement les liens sociaux et les caractères idéologiques des acteurs principaux de l'séconomie dirigée«, mais aussi leur pratique de pouvoir, en particulier dans le contexte de la politique de collaboration. Cette étude ne doit pas être appréhendée de manière exclusivement statique, dans le sens d'une confrontation comparative des deux régimes, mais aussi de manière dynamique, comme analyse de leur influence réciproque tout au long de la période de l'Occupation. Cette influence se manifeste surtout dans le domaine de la politique économique.

L'auteur étudie d'abord les formes spécifiques de l'adaptation stratégique de l'administration militaire allemande aux particularités de la situation en France lors de l'armistice de 1940 ainsi que de la réalité socio-structurelle et institutionnelle de la polycratie nationalsocialiste dans la France occupée, avant d'aborder la question du rôle réel des technocrates et de l'importance de la direction globale de l'économie dans le cadre du programme d'une réorganisation de l'État français. Il s'interroge ensuite sur les expériences de socialisation et de pouvoir de l'élite technocrate française ainsi que sur le rôle des facteurs idéologiques et pragmatiques dans la politique de collaboration de celle-ci. Il aborde le cas singulier du technocrate modèle du régime, Jean Bichelonne, qui alliait une extrême intelligence à une radicalité politique prononcée. Après avoir étudié les convergences franco-allemandes d'intérêts en tant que facteur essentiel des influences réciproques des deux régimes, l'auteur analyse enfin la fonction réelle de la collaboration économique aux yeux des technocrates de l'occupant allemand, collaboration économique qui, après une observation plus précise des pratiques administratives, s'avère fonctionner en sens unique au profit du régime d'occupation.

1945 - 1. Oktober 1946. Amtlicher Text in deutscher Sprache, Bd. XXXIX: Urkunden und anderes Beweismaterial, Nürnberg 1949, S. 391.

${ }^{76}$ Arne RADTKE-DELACOR, Verlängerte Werkbank im Westen: Deutsche Produktionsaufträge als Trumpfkarte der industriellen Kollaboration in Frankreich (1942-1944), in: Stefan MARTENS, Maurice VAïSSE (Hg.), Frankreich und Deutschland im Krieg (November 1942Herbst 1944). Okkupation, Kollaboration, Résistance, Bonn 2000 (Pariser Historische Studien, 55), S. 327-350; DERS., Produire pour le Reich. Les commandes allemandes à l'industrie française (1940-1944), in: Vingtième Siècle. Revue d'histoire 70 (2001) S. 99-115. 